Article

\title{
Analysis of Geometric Properties of Ternary Four-Point Rational Interpolating Subdivision Scheme
}

\author{
Pakeeza Ashraf ${ }^{1}$, Bushra Nawaz ${ }^{1}$, Dumitru Baleanu ${ }^{2,3,4}$ (D), Kottakkaran Sooppy Nisar ${ }^{5, *}$ (D), \\ Abdul Ghaffar $6,7, *$ (D), Muhammad Aqeel Ahmed Khan ${ }^{8}$ and Saima Akram ${ }^{9}$ \\ 1 Department of Mathematics, Government Sadiq College Women University, Bahawalpur 63100, Pakistan; \\ pakeeza@gscwu.edu.pk (P.A.); bushranawaz1208@gmail.com (B.N.) \\ 2 Department of Mathematics, Cankaya University, 06790 Ankara, Turkey; dumitru@cankaya.edu.tr \\ 3 Institute of Space Sciences, 077125 Magurele-Bucharest, Romania \\ 4 Department of Medical Research, China Medical University Hospital, China Medical University, \\ Taichung 40447, Taiwan \\ 5 Department of Mathematics, College of Arts and Sciences, Prince Sattam bin Abdulaziz University, \\ Wadi Aldawaser 11991, Saudi Arabia \\ 6 Informetrics Research Group, Ton Duc Thang University, Ho Chi Minh City 700000, Vietnam \\ 7 Faculty of Mathematics \& Statistics, Ton Duc Thang University, Ho Chi Minh City 700000, Vietnam \\ 8 Department of Mathematics, COMSATS University Islamabad, Lahore Campus, Lahore 54000, Pakistan; \\ maqeelkhan@cuilahore.edu.pk \\ 9 Centre for Advanced Studies in Pure and Applied Mathematics Bahauddin Zakariya University Multan, \\ Multan 66000, Pakistan; saimaakram@bzu.edu.pk \\ * Correspondence: n.sooppy@psau.edu.sa (K.S.N.); abdulghaffar@tdtu.edu.vn (A.G.); \\ Tel.: +966-563-456-976 (K.S.N)
}

Received: 3 February 2020; Accepted: 28 February 2020; Published: 4 March 2020

\begin{abstract}
Shape preservation has been the heart of subdivision schemes (SSs) almost from its origin, and several analyses of SSs have been established. Shape preservation properties are commonly used in SSs and various ways have been discovered to connect smooth curves/surfaces generated by SSs to applied geometry. With an eye on connecting the link between SSs and applied geometry, this paper analyzes the geometric properties of a ternary four-point rational interpolating subdivision scheme. These geometric properties include monotonicity-preservation, convexity-preservation, and curvature of the limit curve. Necessary conditions are derived on parameter and initial control points to ensure monotonicity and convexity preservation of the limit curve of the scheme. Furthermore, we analyze the curvature of the limit curve of the scheme for various choices of the parameter. To support our findings, we also present some examples and their graphical representation.
\end{abstract}

Keywords: Monotonicity-preservation; convexity-preservation; curvature; rational interpolating; subdivision schemes

MSC: 65D17; 65D05; 65U07

\section{Introduction}

Shape preservation has great practical importance in the designing of curves/surfaces tailored to industrial design (e.g., related to car, aeroplane or ship modelling where convexity is imposed by technical and physical conditions as well as by aesthetic requirements). These properties are used in the design of curves or surfaces to predict or control their 'shape' by the shape of the control points, that is, the vertices of a given polygonal arc or polyhedral surface. Efficient methods to 
construct shape-preserving approximations starting from an initial data sequence are shape-preserving subdivision schemes, that is, schemes that preserve the shape for instance, the monotonicity or the convexity of the control points. In the past years, the shape preservation of limit curves has been a reliable topic in research. The main desirable properties for the implementation of smooth curves are monotonicity and convexity preservation.

Yadshalom [1] proposed a class of subdivision scheme having finite support of curve designing which preserved monotonicity. Carnicer and Dahmen [2] examined a characterization of strict convexity preserving subdivision schemes (SS). Dyn et al. [3] introduced interpolatory 4-point SS for curve designing, which preserved convexity. Kuijt and Damme [4] examined interpolating SS on shape preservation for non-uniform data. They also presented sufficient conditions for convexity preservation. Cai [5] introduced four-point ternary interpolating SS which preserved convexity and having a $C^{2}$ limit curve. A convexity preserving binary six-point linear approximating SS was presented by Hao et al. [6]. Mustafa et al. [7] introduced $n$-ary interpolating SS in which convexity preservation of the scheme was also examined. Floater et al. [8] offered monotonicity preservation of input data having conditions that assure differentiability to the limit function of the SS.

The shape-preserving property of ternary SS with the bell-shaped mask was examined by Pitolli [9]. A six-point interpolating ternary SS was presented by Mustafa and Ashraf [10], in which smoothness and differentiability of limit curves were discussed. Further, Siddiqi and Noreen [11] analyzed convexity preservation of six-point ternary interpolating SS. Akram et al. [12] proposed binary 4-point interpolating non-stationary scheme which preserved positivity, monotonicity, and convexity. Wang and $\mathrm{Li}$ [13] offered a five-point binary scheme that preserved convexity.

Mustafa and Bashir [14] dealt with the univariate scheme and its non-tensor product generalization of bivariate SS. The proposed schemes preserved the monotonicity of initial data. Novara and Romani [15] gave the conditions that the free parameter of the interpolating 5-point ternary SS and the vertices of a strictly convex initial polygon needs to ensure the convexity preservation of the limit curve. Asghar et al. [16] examined SS on probability distribution which has high continuity. The family of SS preserved convexity. A class of non-stationary $2 m$-point binary scheme was presented by Ghaffar et al. [17]. They analyzed curvature and torsion of the limit curves of the proposed schemes. Zulkifli et al. [18] discussed the application of new rational bi-cubic Ball function with six parameters in image interpolation, especially for the gray scale image. For more recent work on SS one may refer to References [19-23].

A SS is said to be rational when the coefficients of the scheme are rational numbers. A ternary four-point rational scheme (FPR-scheme) was presented by Peng [24].

The FPR-scheme plots a polygon $F^{j}=\left\{f_{i}^{j}\right\}_{i \in \mathbb{Z}}$ to define polygon $F^{j+1}=\left\{f_{i}^{j+1}\right\}_{i \in \mathbb{Z}}$ by connecting the resultant subdivision rule and having set of control points at level $k(k \geq 0, k \in \mathbb{Z})$.

$$
\left\{\begin{array}{l}
f_{3 i}^{k+1}=f_{i}^{k} \\
f_{3 i+1}^{k+1}=s_{0} f_{i-1}^{k}+s_{1} f_{i}^{k}+s_{2} f_{i+1}^{k}+s_{3} f_{i+2}^{k} \\
f_{3 i+2}^{k+1}=s_{3} f_{i-1}^{k}+s_{2} f_{i}^{k}+s_{1} f_{i+1}^{k}+s_{0} f_{i+2}^{k}
\end{array}\right.
$$

such that

$$
\left\{\begin{array}{l}
s_{0}=\frac{-243 u+2}{27(126 u-1)} \\
s_{1}=\frac{873 u-7}{9(126 u-1)} \\
s_{2}=\frac{129 u-1}{3(126 u-1)} \\
s_{3}=\frac{-135 u+1}{27(126 u-1)}
\end{array}\right.
$$

where the set of non-zero coefficients is called the mask of the scheme and $u$ is shape parameter. The necessary condition for the convergence of SS is 


$$
\sum_{j \in \mathbb{Z}} s_{3 j}=\sum_{j \in \mathbb{Z}} s_{3 j+1}=\sum_{j \in \mathbb{Z}} s_{3 j+2}=1
$$

Combining Equations (1) and (2), the FPR-scheme can be expressed as:

$$
\left\{\begin{array}{l}
f_{3 i}^{k+1}=f_{i}^{k} \\
f_{3 i+1}^{k+1}=\frac{-243 u+2}{27(126 u-1)} f_{i-1}^{k}+\frac{873 u-7}{9(126 u-1)} f_{i}^{k}+\frac{129 u-1}{3(126 u-1)} f_{i+1}^{k}+\frac{-135 u+1}{27(126 u-1)} f_{i+2}^{k} \\
f_{3 i+2}^{k+1}=\frac{-135 u+1}{27(126 u-1)} f_{i-1}^{k}+\frac{129 u-1}{3(126 u-1)} f_{i}^{k}+\frac{873 u-7}{9(126 u-1)} f_{i+1}^{k}+\frac{-243 u+2}{27(126 u-1)} f_{i+2}^{k} .
\end{array}\right.
$$

Using Equation (3), it is evident that (4) is convergent. Peng et al. [24] proved that the FPR-scheme generates $C^{2}$ limit curves when $u \in(-\infty, 0) \cup\left(\frac{1}{86}, \infty\right)$. They also analyzed the fractal behaviour of the scheme.

This motivated us to present the analysis of the geometric properties of the $C^{2}$-continuous ternary scheme which is capable of producing fractal curves. In order to show the performance of the ternary scheme, we analyze the geometric properties such as monotonicity-preservation, convexity-preservation and curvature of the limit curve. Moreover, the limit curves with specific value of shape control parameter $u$ are depicted by significant application of derived conditions on the initial data. The rest of the paper is organized as follows: In Section 2, we present geometric properties of the FPR-scheme. Numerical examples and conclusions are discussed in Section 3.

\section{Geometric Properties of the FPR-Scheme}

In this section, we present the geometric properties of the limit curves generated by the $F P R$-scheme. These geometric properties consist of monotonicity-preservation, convexity preservation and curvature of the limit curves of the geometric properties.

\subsection{Monotonicity Preservation}

Here we discuss the monotonicity preserving property of the $F P R$-scheme. For monotonicity preservation, we consider monotone control polygon since the limiting curve generated by the FPR-scheme preserves the monotonicity of initial data. The monotonicity preservation of the $F P R$-scheme can be obtained by applying the first order divided difference (DD) as $D_{i}^{k}=f_{i+1}^{k}-f_{i}^{k}$. Thus the first order DD-scheme of (4) can be written as

$$
\left\{\begin{array}{l}
D_{3 i}^{k+1}=\frac{1}{9(126 u-1)}\left((243 u-2) D_{i}^{k}+(1026 u-8) D_{i+1}^{k}+(-135 u+1) D_{i+2}^{k}\right), \\
D_{3 i+1}^{k+1}=\frac{1}{9(126 u-1)}\left((-108 u+1) D_{i}^{k}+(1350 u-11) D_{i+1}^{k}+(-108 u+1) D_{i+2}^{k}\right), \\
D_{3 i+2}^{k+1}=\frac{1}{9(126 u-1)}\left((-135 u+1) D_{i}^{k}+(1026 u-8) D_{i+1}^{k}+(243 u-2) D_{i+2}^{k}\right) .
\end{array}\right.
$$

For convenience, we introduce a new parameter $\omega$ in terms of $u$. For this let $\omega=\frac{-108 u+1}{9(126 u-1)}$, so the value of $u$ in terms of $\omega$, can be written as:

$$
u=\frac{1+9 \omega}{9(126 \omega+12)}
$$

By combining Equations (5) and (6) the first order DD-scheme of (4) takes the form

$$
\left\{\begin{array}{l}
D_{3 i}^{k+1}=\left(\frac{1-3 \omega}{6}\right) D_{i}^{k}+(1+\omega) D_{i+1}^{k}-\left(\frac{1+3 \omega}{6}\right) D_{i+2}^{k} \\
D_{3 i+1}^{k+1}=\omega D_{i}^{k}+(1-2 \omega) D_{i+1}^{k}+\omega D_{i+2^{\prime}}^{k} \\
D_{3 i+2}^{k+1}=-\left(\frac{1+3 \omega}{6}\right) D_{i}^{k}+(1+\omega) D_{i+1}^{k}+\left(\frac{1-3 \omega}{6}\right) D_{i+2}^{k} .
\end{array}\right.
$$


Theorem 1. Given a set of initial control points $\left\{f_{i}^{0}\right\}_{i \in \mathbb{Z}}$ which are monotonically increasing, such that $D_{i}^{0} \geq 0$. Let

$$
r_{i}^{k}=\frac{D_{i+1}^{k}}{D_{i}^{k}}, \quad R_{i}^{k}=\max \left\{r_{i}^{k}, \frac{1}{r_{i}^{k}}\right\}, \forall k \geq 0, k \in \mathbb{Z}, i \in \mathbb{Z}
$$

Furthermore, the parameter $\omega$ satisfies $-0.077 \leq \omega<0$. If $\frac{1}{\lambda} \leq R_{i}^{0} \leq \lambda,\left\{f_{i}^{k}\right\}_{i \in \mathbb{Z}}$ is defined by the FPR-scheme (4), then

$$
D_{i}^{k} \geq 0, \quad \frac{1}{\lambda} \leq R_{i}^{k} \leq \lambda \forall k \geq 0, k \in \mathbb{Z}, i \in \mathbb{Z} .
$$

Thus, the FPR-scheme preserves monotonicity for initial monotone data.

Proof. To prove (8), we use mathematical induction.

By assumption it is clear that (8) holds for $k=0$. Suppose that (8) holds for $k \geq 1$, next we verify that it also holds for $k+1$.

In order to show that (8) is true for $k+1$, we first prove that $D_{i}^{k+1} \geq 0, \forall k \geq 0, k \in \mathbb{Z}, i \in \mathbb{Z}$. As

$$
\begin{aligned}
D_{3 i}^{k+1} & =\left(\frac{1-3 \omega}{6}\right) D_{i}^{k}+(1+\omega) D_{i+1}^{k}-\left(\frac{1+3 \omega}{6}\right) D_{i+2}^{k} \\
& =D_{i+1}^{k}\left(\left(\frac{1-3 \omega}{6}\right) \frac{1}{r_{i}^{k}}+1+\omega-\left(\frac{1+3 \omega}{6}\right) r_{i+1}^{k}\right) \\
& \geq D_{i+1}^{k}\left(\left(\frac{1-3 \omega}{6}\right) \frac{1}{\lambda}+1+\omega-\left(\frac{1+3 \omega}{6}\right) \lambda\right) .
\end{aligned}
$$

For convenience put $\lambda=-\frac{2 \omega}{1-2 \omega}$, we get

$$
D_{3 i}^{k+1} \geq D_{i+1}^{k}\left(\frac{24 \omega^{2}-19 \omega+1}{12 \omega(-1+2 \omega)}\right) \geq 0 .
$$

Thus

$$
D_{3 i}^{k+1} \geq 0
$$

Now consider

$$
\begin{aligned}
D_{3 i+1}^{k+1} & =\omega D_{i}^{k}+(1-2 \omega) D_{i+1}^{k}+\omega D_{i+2}^{k} \\
& =D_{i+1}^{k}\left(\omega \frac{1}{r_{i}^{k}}+1-2 \omega+\omega r_{i+1}^{k}\right)
\end{aligned}
$$

Since $\frac{1}{\lambda} \leq R_{i}^{k} \leq \lambda$, so $r_{i}^{k} \geq \frac{1}{\lambda}$ and $\frac{1}{r_{i}^{k}} \geq \frac{1}{\lambda} \forall k \geq 0, k \in \mathbb{Z}, i \in \mathbb{Z}$, then we have

$$
\begin{aligned}
D_{3 i+1}^{k+1} & \geq D_{i+1}^{k}\left(\omega \frac{1}{\lambda}+1-2 \omega+\omega \frac{1}{\lambda}\right) \\
& =D_{i+1}^{k}\left(\frac{(2 \omega-2 \omega)(1-2 \omega)}{-2 \omega}\right) \geq 0 .
\end{aligned}
$$

Thus

$$
D_{3 i+1}^{k+1} \geq 0
$$


Now consider

$$
\begin{aligned}
D_{3 i+2}^{k+1} & =-\left(\frac{1+3 \omega}{6}\right) D_{i}^{k}+(1+\omega) D_{i+1}^{k}+\left(\frac{1-3 \omega}{6}\right) D_{i+2}^{k} \\
& =D_{i+1}^{k}\left(-\left(\frac{1+3 \omega}{6}\right) \frac{1}{r_{i}^{k}}+1+\omega+\left(\frac{1-3 \omega}{6}\right) r_{i+1}^{k}\right) \\
& \geq D_{i+1}^{k}\left(-\left(\frac{1+3 \omega}{6}\right) \lambda+1+\omega+\left(\frac{1-3 \omega}{6}\right) \frac{1}{\lambda}\right) \\
& \geq D_{i+1}^{k}\left(\frac{24 \omega^{2}-19 \omega+1}{12 \omega(-1+2 \omega)}\right) \geq 0 .
\end{aligned}
$$

Therefore

$$
D_{3 i+2}^{k+1} \geq 0
$$

By combining Equations (9)-(11), we have $D_{i}^{k+1} \geq 0$ for $\forall k \geq 0, k \in \mathbb{Z}, i \in \mathbb{Z}$. Thus $D_{i}^{k} \geq 0$. Further we prove $\frac{1}{\lambda} \leq R_{i}^{k+1} \leq \lambda, \forall k \geq 0, k \in \mathbb{Z}, i \in \mathbb{Z}$.

Since

$$
\begin{aligned}
r_{3 i}^{k+1} & =\frac{D_{3 i+1}^{k+1}}{D_{3 i}^{k+1}}=\frac{\omega D_{i}^{k}+(1-2 \omega) D_{i+1}^{k}+\omega D_{i+2}^{k}}{\left(\frac{1-3 \omega}{6}\right) D_{i}^{k}+(1+\omega) D_{i+1}^{k}-\left(\frac{1+3 \omega}{6}\right) D_{i+2}^{k}} \\
& =\frac{D_{i+1}^{k}\left(\omega \frac{1}{r_{i}^{k}}+1-2 \omega+\omega r_{i+1}^{k}\right)}{D_{i+1}^{k}\left(\left(\frac{1-3 \omega}{6}\right) \frac{1}{r_{i}^{k}}+1+\omega-\left(\frac{1+3 \omega}{6}\right) r_{i+1}^{k}\right)} .
\end{aligned}
$$

Now consider

$$
\begin{aligned}
r_{3 i}^{k+1}-\lambda & =\frac{\omega \frac{1}{r_{i}^{k}}+1-2 \omega+\omega r_{i+1}^{k}}{\left(\frac{1-3 \omega}{6}\right) \frac{1}{r_{i}^{k}}+1+\omega-\left(\frac{1+3 \omega}{6}\right) r_{i+1}^{k}}-\lambda \\
& =\frac{\omega \frac{1}{r_{i}^{k}}+1-2 \omega+\omega r_{i+1}^{k}-\left(\frac{1-3 \omega}{6}\right) \lambda \frac{1}{r_{i}^{k}}-(1+\omega) \lambda+\left(\frac{1+3 \omega}{6}\right) \lambda r_{i+1}^{k}}{\left(\frac{1-3 \omega}{6}\right) \frac{1}{r_{i}^{k}}+1+\omega-\left(\frac{1+3 \omega}{6}\right) r_{i+1}^{k}} .
\end{aligned}
$$

By Equation (9), the denominator of the above equation is greater and equal to zero, and the numerator $A$ satisfies

$$
\begin{aligned}
A & \leq \omega \frac{1}{r_{i}^{k}}+1-2 \omega+\omega r_{i+1}^{k}-\left(\frac{1-3 \omega}{6}\right) \lambda \frac{1}{r_{i}^{k}}-(1+\omega) \lambda+\left(\frac{1+3 \omega}{6}\right) \lambda r_{i+1}^{k} \\
& \leq-\left(-\omega+\left(\frac{1-3 \omega}{6}\right) \lambda\right) \frac{1}{\lambda}+1-2 \omega-(1+\omega) \lambda-\left(-\omega-\left(\frac{1+3 \omega}{6}\right) \lambda\right) \frac{1}{\lambda} \\
& \leq \frac{3 \omega}{2 \omega-1} \leq 0 .
\end{aligned}
$$

Therefore, $r_{3 i}^{k+1} \leq \lambda$. 
Further we have

$$
\begin{aligned}
r_{3 i+1}^{k+1} & =\frac{D_{3 i+2}^{k+1}}{D_{3 i+1}^{k+1}}=\frac{-\left(\frac{1+3 \omega}{6}\right) D_{i}^{k}+(1+\omega) D_{i+1}^{k}+\left(\frac{1-3 \omega}{6}\right) D_{i+2}^{k}}{\omega D_{i}^{k}+(1-2 \omega) D_{i+1}^{k}+\omega D_{i+2}^{k}} \\
& =\frac{D_{i+1}^{k}\left(-\left(\frac{1+3 \omega}{6}\right) \frac{1}{r_{i}^{k}}+1+\omega+\left(\frac{1-3 \omega}{6}\right) r_{i+1}^{k}\right)}{D_{i+1}^{k}\left(\omega \frac{1}{r_{i}^{k}}+1-2 \omega+\omega r_{i+1}^{k}\right)} .
\end{aligned}
$$

Now consider

$$
\begin{aligned}
r_{3 i+1}^{k+1}-\lambda & =\frac{-\left(\frac{1+3 \omega}{6}\right) \frac{1}{r_{i}^{k}}+1+\omega+\left(\frac{1-3 \omega}{6}\right) r_{i+1}^{k}}{\omega \frac{1}{r_{i}^{k}}+1-2 \omega+\omega r_{i+1}^{k}}-\lambda \\
& =\frac{-\left(\frac{1+3 \omega}{6}\right) \frac{1}{r_{i}^{k}}+1+\omega+\left(\frac{1-3 \omega}{6}\right) r_{i+1}^{k}-\omega \lambda \frac{1}{r_{i}^{k}}-(1-2 \omega) \lambda-\omega \lambda r_{i+1}^{k}}{\omega \frac{1}{r_{i}^{k}}+1-2 \omega+\omega r_{i+1}^{k}} .
\end{aligned}
$$

By Equation (10), the denominator of the above equation is greater and equal to zero, and the numerator $B$ satisfies

$$
\begin{aligned}
B & \leq-\left(\frac{1+3 \omega}{6}\right) \frac{1}{r_{i}^{k}}+1+\omega+\left(\frac{1-3 \omega}{6}\right) r_{i+1}^{k}-\omega \lambda \frac{1}{r_{i}^{k}}-(1-2 \omega) \lambda-\omega \lambda r_{i+1}^{k} \\
& \leq-\left(\frac{1+3 \omega}{6}\right) \frac{1}{\lambda}+1+\omega+\left(\frac{1-3 \omega}{6}\right) \lambda-\omega \lambda \frac{1}{\lambda}-(1-2 \omega) \lambda-\omega \lambda \frac{1}{\lambda} \\
& \leq \frac{24 \omega^{2}-11 \omega-1}{12\left(2 \omega^{2}-\omega\right)} \leq 0 .
\end{aligned}
$$

Thus $r_{3 i+1}^{k+1} \leq \lambda$.

Similarly

$$
\begin{aligned}
r_{3 i+2}^{k+1} & =\frac{D_{3 i+3}^{k+1}}{D_{3 i+2}^{k+1}}=\frac{\left(\frac{1-3 \omega}{6}\right) D_{i+1}^{k}+(1+\omega) D_{i+2}^{k}-\left(\frac{1+3 \omega}{6}\right) D_{i+3}^{k}}{-\left(\frac{1+3 \omega}{6}\right)(1+\omega) D_{i+1}^{k}+\left(\frac{1-3 \omega}{6}\right) D_{i+2}^{k}} \\
& =\frac{D_{i+1}^{k}\left(\left(\frac{1-3 \omega}{6}\right)+(1+\omega) r_{i+1}^{k}-\left(\frac{1+3 \omega}{6}\right) r_{i+1}^{k} r_{i+2}^{k}\right)}{D_{i+1}^{k}\left(-\left(\frac{1+3 \omega}{6}\right) \frac{1}{r_{i}^{k}}+1+\omega+\left(\frac{1-3 \omega}{6}\right) r_{i+1}^{k}\right)} .
\end{aligned}
$$

So

$$
\begin{aligned}
r_{3 i+2}^{k+1}-\lambda & =\frac{\left(\frac{1-3 \omega}{6}\right)+(1+\omega) r_{i+1}^{k}-\left(\frac{1+3 \omega}{6}\right) r_{i+1}^{k} r_{i+2}^{k}}{-\left(\frac{1+3 \omega}{6}\right) \frac{1}{r_{i}^{k}}+1+\omega+\left(\frac{1-3 \omega}{6}\right) r_{i+1}^{k}}-\lambda \\
& =\frac{\left(\frac{1-3 \omega}{6}\right)+(1+\omega) r_{i+1}^{k}-\left(\frac{1+3 \omega}{6}\right) r_{i+1}^{k} r_{i+2}^{k}+\left(\frac{1+3 \omega}{6}\right) \lambda \frac{1}{r_{i}^{k}}-(1+\omega) \lambda-\left(\frac{1-3 \omega}{6}\right) \lambda r_{i+1}^{k}}{-\left(\frac{1+3 \omega}{6}\right) \frac{1}{r_{i}^{k}}+1+\omega+\left(\frac{1-3 \omega}{6}\right) r_{i+1}^{k}} .
\end{aligned}
$$

By Equation (11), the denominator of the above equation is greater and equal to zero, and the numerator $C$ satisfies 


$$
\begin{aligned}
C & \leq\left(\frac{1-3 \omega}{6}\right)+\left((1+\omega)-\left(\frac{1+3 \omega}{6}\right) \frac{1}{\lambda}\right) \lambda+\left(\frac{1+3 \omega}{6}\right) \lambda^{2}-(1+\omega) \lambda-\left(\frac{1-3 \omega}{6}\right) \lambda \frac{1}{\lambda} \\
& \leq\left(\frac{1+3 \omega}{6}\right)\left(-\frac{2 \omega}{1-2 \omega}\right)^{2}-\left(\frac{1+3 \omega}{6}\right) \\
& \leq \frac{(3 \omega+1)(4 \omega-1)}{6(-1+2 \omega)^{2}} \leq 0 .
\end{aligned}
$$

Therefore $r_{3 i+2}^{k+1} \leq \lambda$.

By following same steps, we can also prove $\frac{1}{r_{3 i}^{k+1}} \leq \lambda, \frac{1}{r_{3 i+1}^{k+1}} \leq \lambda$ and $\frac{1}{r_{3 i+2}^{k+1}} \leq \lambda$. Therefore, $\frac{1}{\lambda} \leq R_{i}^{k+1} \leq \lambda$ and by induction, we have $\frac{1}{\lambda} \leq R_{i}^{k} \leq \lambda, \forall k \in \mathbb{Z}, i \in \mathbb{Z}$.

This completes the proof.

\subsection{Convexity Preservation}

Now we discuss the convexity preservation of the FPR-scheme. For convexity preservation, we consider convex control polygon so the limiting curve generated by the $F P R$-scheme preserves convexity of initial data. The convexity preservation of the FPR-scheme can be obtained by applying second order DD as $P_{i}^{k}=3^{2 k}\left(f_{i-1}^{k}-2 f_{i}^{k}+f_{i+1}^{k}\right)$.

$$
\left\{\begin{array}{l}
P_{3 i}^{k+1}=\frac{117 u-1}{126 u-1} P_{i}^{k}+\frac{9 u}{126 u-1} P_{i+1}^{k} \\
P_{3 i+1}^{k+1}=\frac{9 u}{126 u-1} P_{i}^{k}+\frac{117 u-1}{126 u-1} P_{i+1}^{k} \\
P_{3 i+2}^{k+1}=\frac{-135 u+1}{3(126 u-1)} P_{i}^{k}+\frac{648 u-5}{3(126 u-1)} P_{i+1}^{k}+\frac{-135 u+1}{3(126 u-1)} P_{i+2}^{k}
\end{array}\right.
$$

For convenience, we introduce a new parameter $\sigma$ instead of $u$. For this let $\sigma=\frac{1-135 u}{3(126 u-1)}$ and $u \neq \frac{1}{126}$, so the value of $u$ in terms of $\sigma$ can be expressed as

$$
u=\frac{1+3 \sigma}{378 \sigma+135}, \quad \sigma \neq-\frac{135}{378}
$$

By combining Equations (12) and (13), second order DD-scheme takes the form

$$
\left\{\begin{array}{l}
P_{3 i}^{k+1}=(2+3 \sigma) P_{i}^{k}-(1+3 \sigma) P_{i+1}^{k} \\
P_{3 i+1}^{k+1}=-(1+3 \sigma) P_{i}^{k}+(2+3 \sigma) P_{i+1}^{k} \\
P_{3 i+2}^{k+1}=\sigma P_{i}^{k}+(1-2 \sigma) P_{i+1}^{k}+\sigma P_{i+2}^{k}
\end{array}\right.
$$

Theorem 2. Given a set of initial control points $\left\{f_{i}^{0}\right\}_{i \in \mathbb{Z}}$ which are convex, such that $P_{i}^{0} \geq 0, \forall i \in \mathbb{Z}$, denote $q_{i}^{k}=\frac{P_{i+1}^{k}}{P_{i}^{k}}, Q_{i}^{k}=\max \left\{q_{i}^{k}, \frac{1}{q_{i}^{k}}\right\}, \forall k \geq 0, k \in \mathbb{Z}, i \in \mathbb{Z}$. Furthermore, the parameter $\sigma$ satisfies $-0.33<\sigma \leq-0.20$. If $\frac{1}{\mu} \leq Q_{i}^{0} \leq \mu,\left\{f_{i}^{k}\right\}$ is defined by the FPR-scheme (4), then

$$
P_{i}^{k} \geq 0, \frac{1}{\mu} \leq Q_{i}^{k} \leq \mu \forall k \geq 0, k \in \mathbb{Z}, i \in \mathbb{Z} .
$$

Thus, the FPR-scheme preserves convexity for initial convex data.

Proof. To prove (15), we use mathematical induction.

By assumption it is clear that (15) holds for $k=0$. Assume that (15) also holds for $k \geq 1$, next we verify that it also holds for $k+1$.

In order to show that (15) is true for $k+1$, we first show that $P_{i}^{k+1} \geq 0, \forall k \geq 0, k \in \mathbb{Z}, i \in \mathbb{Z}$. 
As

$$
\begin{aligned}
P_{3 i}^{k+1} & =(2+3 \sigma) P_{i}^{k}-(1+3 \sigma) P_{i+1}^{k} \\
& =P_{i+1}^{k}\left((2+3 \sigma) \frac{1}{q_{i}^{k}}-1-3 \sigma\right) \\
& \geq P_{i+1}^{k}\left((2+3 \sigma) \frac{1}{\mu}-1-3 \sigma\right) .
\end{aligned}
$$

For convenience put $\mu=\frac{2+3 \sigma}{1+3 \sigma}, \sigma \neq-\frac{1}{3}$, we get

$$
P_{3 i}^{k+1} \geq P_{i+1}^{k}(1+3 \sigma-1-3 \sigma) \geq 0 .
$$

Thus

$$
P_{3 i}^{k+1} \geq 0
$$

Now consider

$$
\begin{aligned}
P_{3 i+1}^{k+1} & =-(1+3 \sigma) P_{i}^{k}+(2+3 \sigma) P_{i+1}^{k} \\
& =P_{i+1}^{k}\left(-(1+3 \sigma) \frac{1}{q_{i}^{k}}+2+3 \sigma\right) \\
& \geq P_{i+1}^{k}(-(1+3 \sigma) \mu+2+3 \sigma) \\
& \geq P_{i+1}^{k}(-2-3 \sigma+2+3 \sigma) \geq 0 .
\end{aligned}
$$

Thus

$$
P_{3 i+1}^{k+1} \geq 0
$$

Now consider

$$
\begin{aligned}
P_{3 i+2}^{k+1} & =\sigma P_{i}^{k}+(1-2 \sigma) P_{i+1}^{k}+\sigma P_{i+2}^{k} \\
& =P_{i+1}^{k}\left(\sigma \frac{1}{q_{i}^{k}}+(1-2 \sigma)+\sigma q_{i+1}^{k}\right) \\
& \geq P_{i+1}^{k}\left(\frac{2 \sigma+(1-2 \sigma) \mu}{\mu}\right) \\
& \geq P_{i+1}^{k}\left(\frac{2+\sigma}{2+3 \sigma}\right) \geq 0 .
\end{aligned}
$$

Therefore

$$
P_{3 i+2}^{k+1} \geq 0
$$

So by combining Equations (16)-(18), we have $P_{i}^{k+1} \geq 0, \forall i \in \mathbb{Z}$. Thus $P_{i}^{k} \geq 0, \forall k \geq 0, k \in \mathbb{Z}, i \in$ $\mathbb{Z}$. Now we will prove $\frac{1}{\mu} \leq Q_{i}^{k} \leq \mu, \forall i \in \mathbb{Z}, k \geq 0$. 
Consider

$$
\begin{aligned}
q_{3 i}^{k+1} & =\frac{P_{3 i+1}^{k+1}}{P_{3 i}^{k+1}}=\frac{-(1+3 \sigma) P_{i}^{k}+(2+3 \sigma) P_{i+1}^{k}}{(2+3 \sigma) P_{i}^{k}-(1+3 \sigma) P_{i+1}^{k}} \\
& =\frac{P_{i+1}^{k}\left(-(1+3 \sigma) \frac{1}{q_{i}^{k}}+2+3 \sigma\right)}{P_{i+1}^{k}\left((2+3 \sigma) \frac{1}{q_{i}^{k}}-1-3 \sigma\right)} .
\end{aligned}
$$

Further, we have

$$
\begin{aligned}
q_{3 i}^{k+1}-\mu & =\frac{-(1+3 \sigma) \frac{1}{q_{i}^{k}}+2+3 \sigma}{(2+3 \sigma) \frac{1}{q_{i}^{k}}-1-3 \sigma}-\mu \\
& =\frac{-(1+3 \sigma) \frac{1}{q_{i}^{k}}+2+3 \sigma-(2+3 \sigma) \mu \frac{1}{q_{i}^{k}}+(1+3 \sigma) \mu}{(2+3 \sigma) \frac{1}{q_{i}^{k}}-1-3 \sigma}
\end{aligned}
$$

By Equation (16), the denominator of the above equation is greater and equal to zero, and the numerator $D$ satisfies

$$
\begin{aligned}
D & \leq(-(1+3 \sigma)-(2+3 \sigma) \mu) \frac{1}{q_{i}^{k}}+2+3 \sigma+(1+3 \sigma) \mu \\
& \leq(-(1+3 \sigma)-(2+3 \sigma) \mu) \mu+2+3 \sigma+(1+3 \sigma) \mu \\
& \leq \frac{-3((3 \sigma+2)(2 \sigma+1))}{(1+3 \sigma)^{2}} \leq 0 .
\end{aligned}
$$

Therefore, $q_{3 i}^{k+1} \leq \mu$.

Similarly

$$
\begin{aligned}
q_{3 i+1}^{k+1} & =\frac{P_{3 i+2}^{k+1}}{P_{3 i+1}^{k+1}}=\frac{\sigma P_{i}^{k}+(1-2 \sigma) P_{i+1}^{k}+\sigma P_{i+2}^{k}}{-(1+3 \sigma) P_{i}^{k}+(2+3 \sigma) P_{i+1}^{k}} \\
& =\frac{P_{i+1}^{k}\left(\sigma \frac{1}{q_{i}^{k}}+1-2 \sigma+\sigma q_{i+1}^{k}\right)}{P_{i+1}^{k}\left(-(1+3 \sigma) \frac{1}{q_{i}^{k}}+2+3 \sigma\right)} .
\end{aligned}
$$

Since

$$
\begin{aligned}
q_{3 i+1}^{k+1}-\mu & =\frac{\sigma \frac{1}{q_{i}^{k}}+1-2 \omega+\sigma q_{i+1}^{k}}{-(1+3 \sigma) \frac{1}{q_{i}^{k}}+2+3 \sigma}-\mu \\
& =\frac{\sigma \frac{1}{q_{i}^{k}}+1-2 \omega+\sigma q_{i+1}^{k}+(1+3 \sigma) \mu \frac{1}{q_{i}^{k}}-(2+3 \sigma) \mu}{-(1+3 \sigma) \frac{1}{q_{i}^{k}}+2+3 \sigma} .
\end{aligned}
$$

By Equation (17), the denominator of the above equation is greater than and equal to zero, and the numerator $E$ satisfies 


$$
\begin{aligned}
E & \leq \sigma \mu+1-2 \omega+\sigma \mu+(1+3 \sigma) \mu^{2}-(2+3 \sigma) \mu \\
& \leq(1+3 \sigma)\left(\frac{2+3 \sigma}{1+3 \sigma}\right)^{2}-(2+\sigma)\left(\frac{2+3 \sigma}{1+3 \sigma}\right)+1-2 \sigma \\
& \leq \frac{1+5 \sigma}{1+3 \sigma} \leq 0 .
\end{aligned}
$$

Thus, $q_{3 i+1}^{k+1} \leq \mu$.

Also

$$
\begin{aligned}
q_{3 i+2}^{k+1} & =\frac{P_{3 i+3}^{k+1}}{P_{3 i+2}^{k+1}}=\frac{(2+3 \sigma) P_{i+1}^{k}-(1+3 \sigma) P_{i+2}^{k}}{\sigma P_{i}^{k}+(1-2 \sigma) P_{i+1}^{k}+\sigma P_{i+2}^{k}} \\
& =\frac{P_{i+1}^{k}\left(2+3 \sigma-(1+3 \sigma) q_{i+1}^{k}\right)}{P_{i+1}^{k}\left(\sigma \frac{1}{q_{i}^{k}}+1-2 \sigma+\sigma q_{i+1}^{k}\right)}
\end{aligned}
$$

it follows

$$
\begin{aligned}
q_{3 i+2}^{k+1}-\mu & =\frac{2+3 \sigma-(1+3 \sigma) q_{i+1}^{k}}{\sigma \frac{1}{q_{i}^{k}}+1-2 \sigma+\sigma q_{i+1}^{k}}-\mu \\
& =\frac{2+3 \sigma-(1+3 \sigma) q_{i+1}^{k}-\sigma \mu \frac{1}{q_{i}^{k}}-(1-2 \sigma) \mu-\sigma \mu q_{i+1}^{k}}{\sigma \frac{1}{q_{i}^{k}}+1-2 \sigma+\sigma q_{i+1}^{k}} .
\end{aligned}
$$

By Equation (18), the denominator of the above equation is greater than and equal to zero, and the numerator $F$ satisfies

$$
\begin{aligned}
F & \leq 2+3 \sigma-(1+3 \sigma) \frac{1}{\mu}-\sigma \mu \frac{1}{\mu}-(1-2 \sigma) \mu-\sigma \mu \frac{1}{\mu} \\
& =2+\sigma-(1+3 \sigma) \frac{1}{\mu}-(1-2 \sigma) \mu \\
& =2+\sigma-(1+3 \sigma)\left(\frac{1+3 \sigma}{2+3 \sigma}\right)-(1-2 \sigma)\left(\frac{2+3 \sigma}{1+3 \sigma}\right) \\
& =\frac{-1+7 \sigma+15 \sigma^{2}}{(2+3 \sigma)(1+3 \sigma)} \leq 0 .
\end{aligned}
$$

Therefore, $q_{3 i+2}^{k+1} \leq \mu$.

By following same steps we can also prove $\frac{1}{q_{3 i}^{k+1}} \leq \mu, \frac{1}{q_{3 i+1}^{k+1}} \leq \mu$ and $\frac{1}{q_{3 i+2}^{k+1}} \leq \mu$. Therefore, $\frac{1}{\mu} \leq Q_{i}^{k+1} \leq \mu$, and by induction, we have $\frac{1}{\mu} \leq Q_{i}^{k} \leq \mu, \forall k \in \mathbb{Z}, i \in \mathbb{Z}$.

This completes the proof.

\subsection{Curvature}

In mathematics, curvature is any of several strongly related concepts in geometry. Intuitively, the curvature is the amount by which a curve deviates from being a straight line, or a surface deviates from being a plane. Curvature is very important property not only in continuous geometry but also in network applications; see Reference [25].

The quality of SS can be measured quantitatively by finding curvature, as functions of cumulative chord length. We use the method described in Reference [17] to determine the curvature and numerical simulations are provided to illustrate the effects of various choices of shape parameter on curvature. 
Since the FPR-scheme is parametric, it is obvious to see the results for various choices of the parameter $u$. Here we measure the curvature of limit curves of the FPR-scheme for various choices of $u$. In Figures 1 and 2, we consider a control polygon of a circle. The shape of the circle is obtained by applying an FPR-scheme five times on this initial control polygon for various choices of parameter $u$. These limit curves are shown in Figures $1 \mathrm{a}, \mathrm{b}$ and $2 \mathrm{a}, \mathrm{b}$, while their corresponding curvatures are shown in Figures 1c,d and 2c,d. In Figures 3 and 4, we consider a star-shaped closed control polygon. The limit curves after applying the FPR-scheme five times on this initial control polygon for various choices of parameter $u$ is shown in Figures $3 a, b$ and $4 a, b$, while their corresponding curvatures are shown in Figures 1c,d and 2c,d. In Figures 5 and 6, we consider an open control polygon that presents the basic limit function of the scheme. The limit curves after applying the FPR-scheme five times on this initial control polygon for various choices of parameter $u$ is shown in Figures $5 a, b$ and $6 a, b$, while their corresponding curvatures are shown in Figures 5c,d and $6 c, d$.

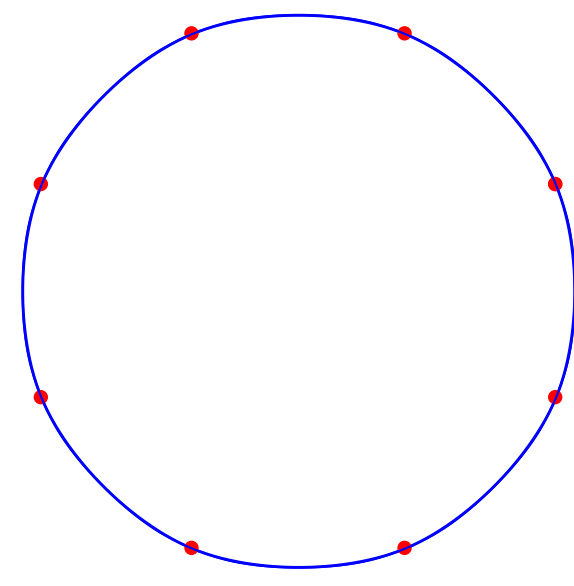

(a)

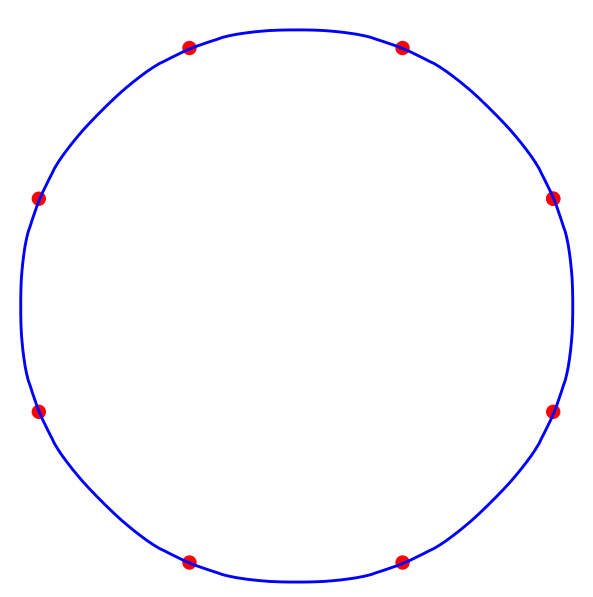

(b)

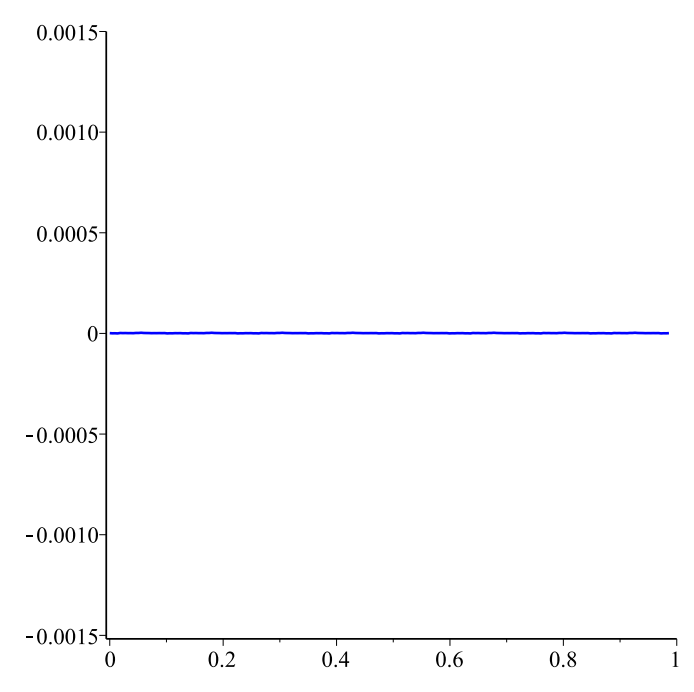

(c)

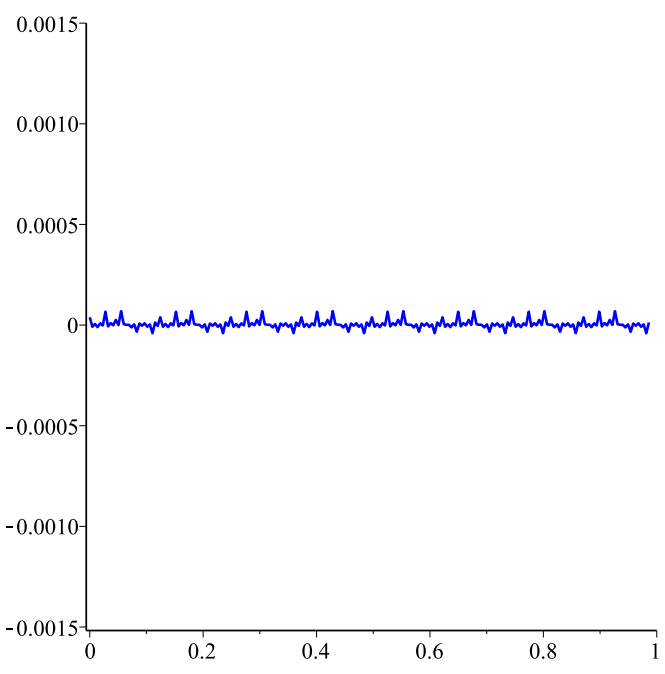

(d)

Figure 1. Results for various choices of the parameter $u$ are shown on the left together with their corresponding curvature on the right. From top to bottom: $u=0$ and 0.0074. (a) Limit curve at $u=0$; (b) Limit curve at $u=0.0074$; (c) Curvature plot of (a); (d) Curvature plot of (b). 


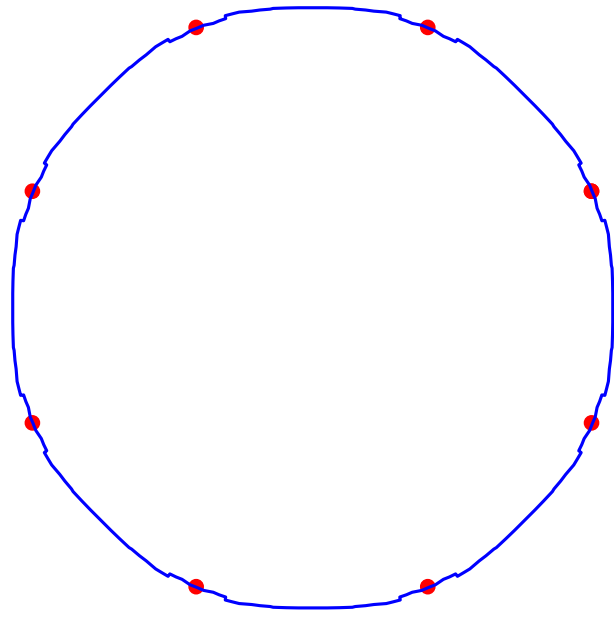

(a)

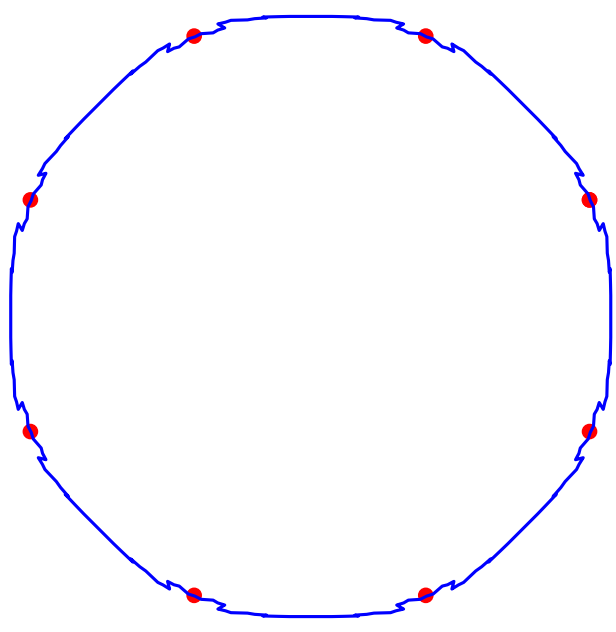

(b)

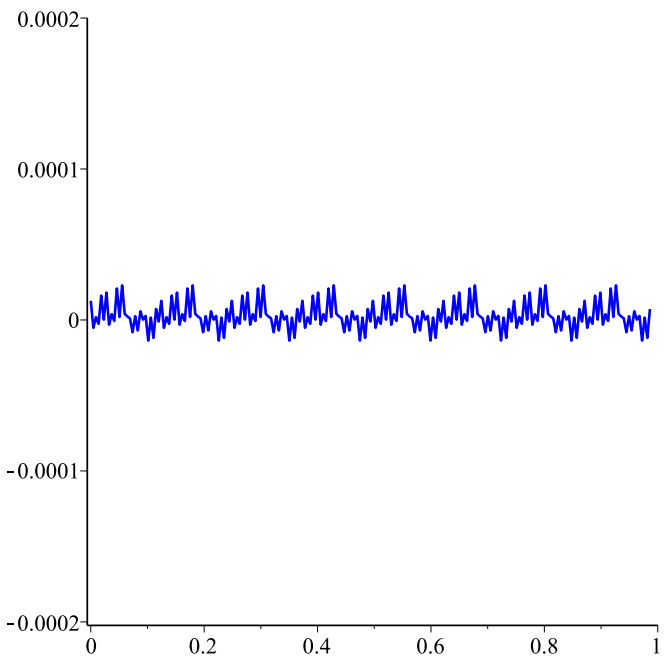

(c)

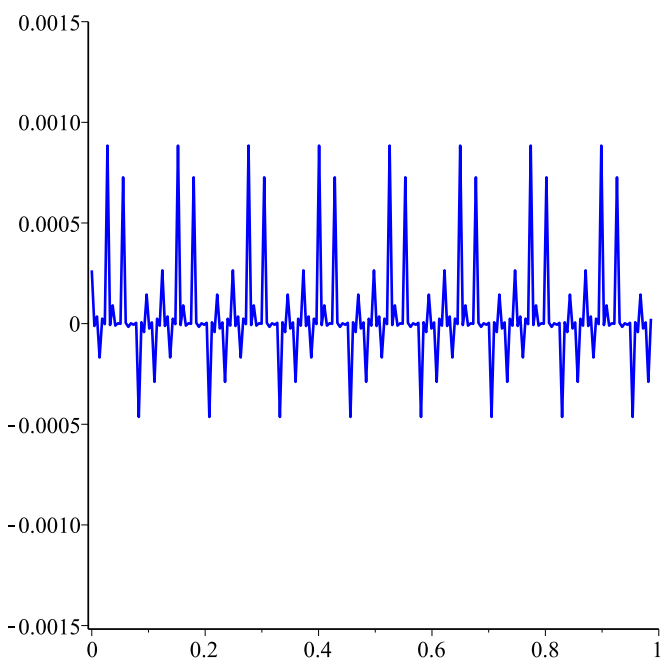

(d)

Figure 2. Results for various choices of the parameter $u$ are shown on the left together with their corresponding curvature on the right. From top to bottom: $u=0.0076$ and 0.0077 . (a) Limit curve at $u=0.0076$; (b) Limit curve at $u=0.0077$; (c) Curvature plot of (a); (d) Curvature plot of (b). 


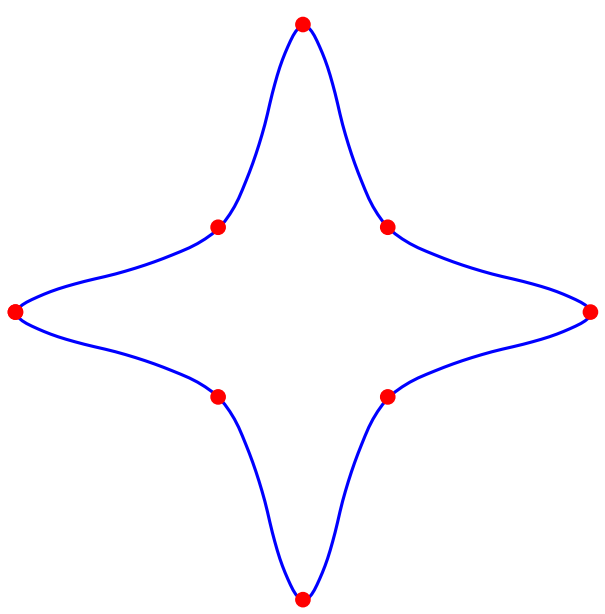

(a)

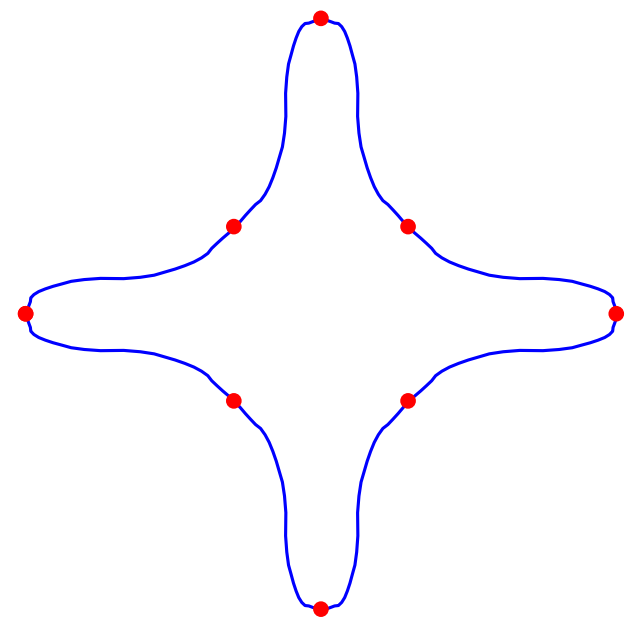

(b)

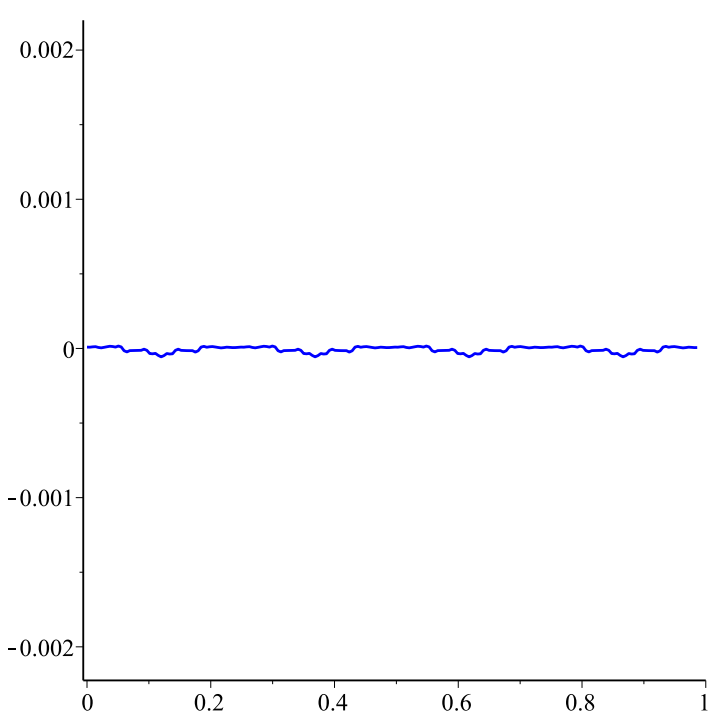

(c)

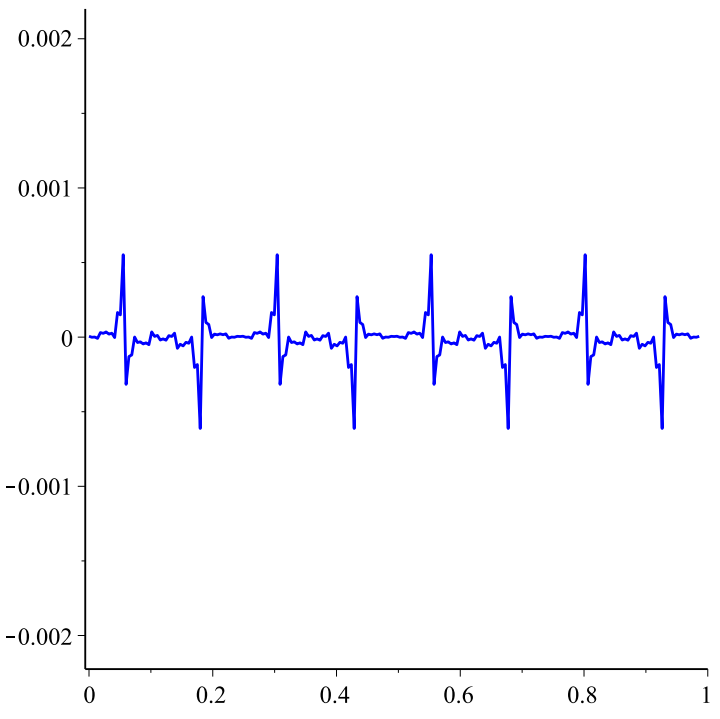

(d)

Figure 3. Results for various choices of the parameter $u$ are shown on the left together with their corresponding curvature on the right. From top to bottom: $u=0$ and 0.0074. (a) Limit curve at $u=0$; (b) Limit curve at $u=0.0074$; (c) Curvature plot of (a); (d) Curvature plot of (b). 


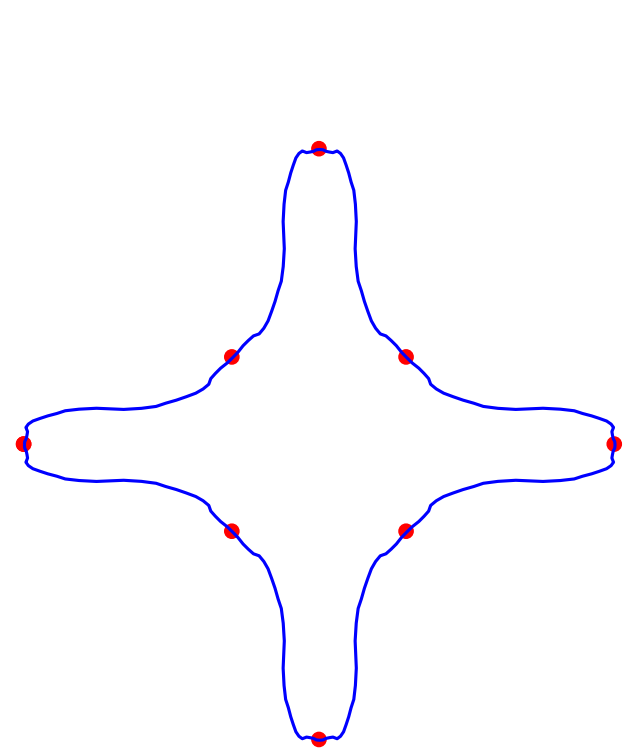

(a)

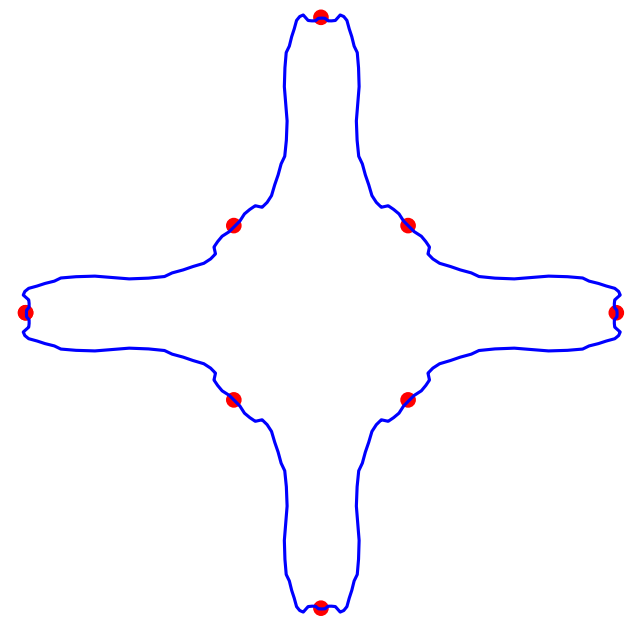

(b)

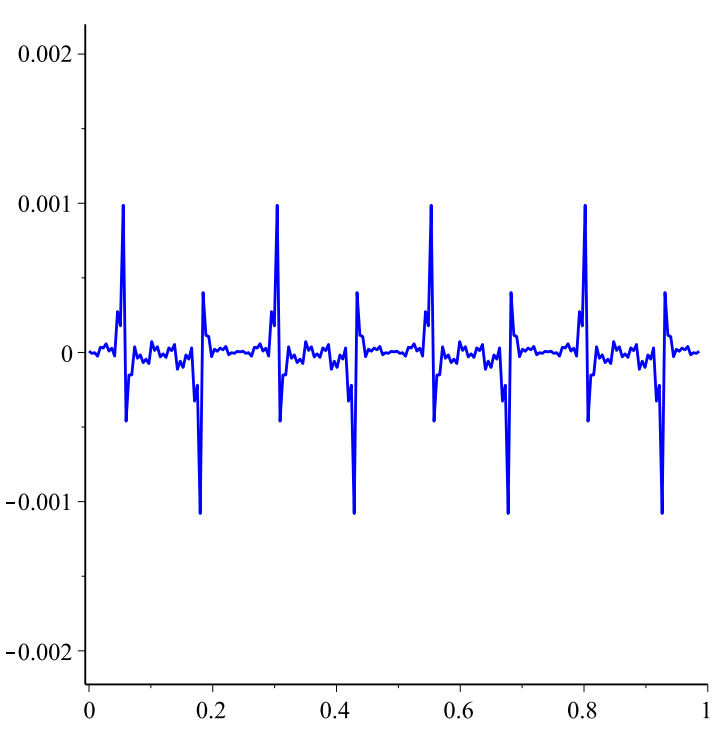

(c)

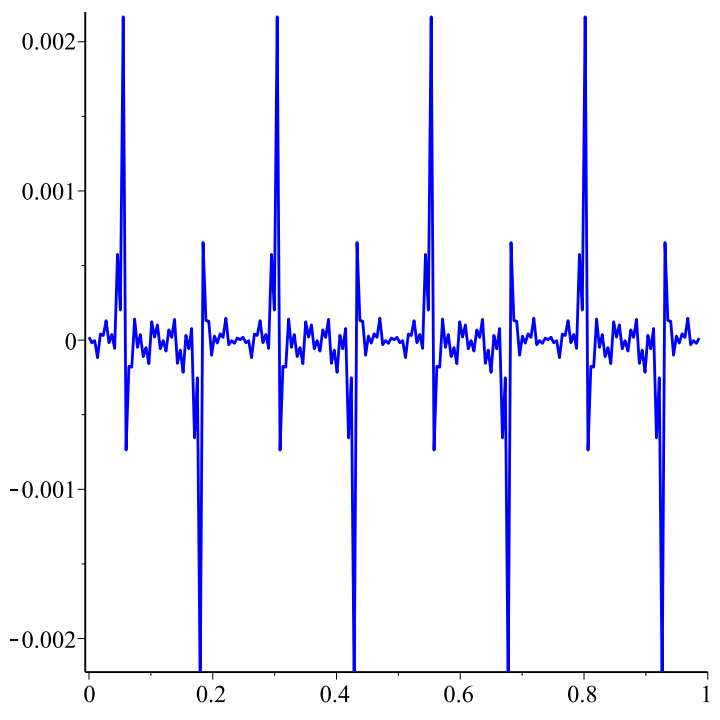

(d)

Figure 4. Results for various choices of the parameter $u$ are shown on the left together with their corresponding curvature on the right. From top to bottom: $u=0.0076$ and 0.0077. (a) Limit curve at $u=0.0076$; (b) Limit curve at $u=0.0077$; (c) Curvature plot of (a); (d) Curvature plot of (b). 


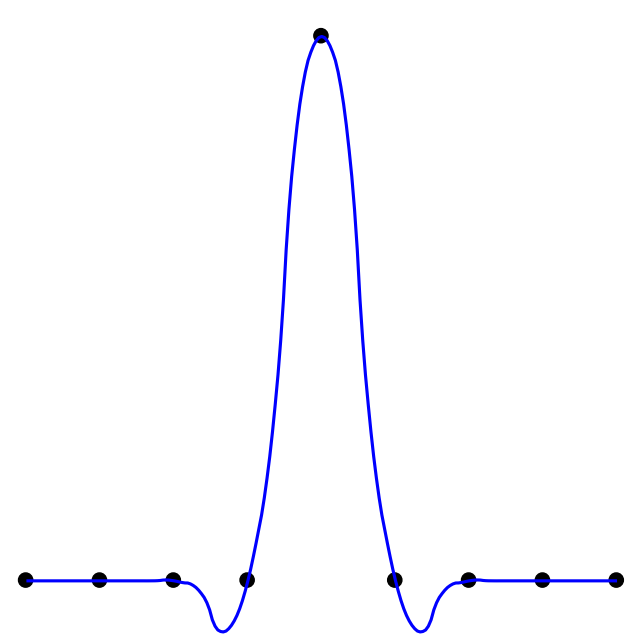

(a)

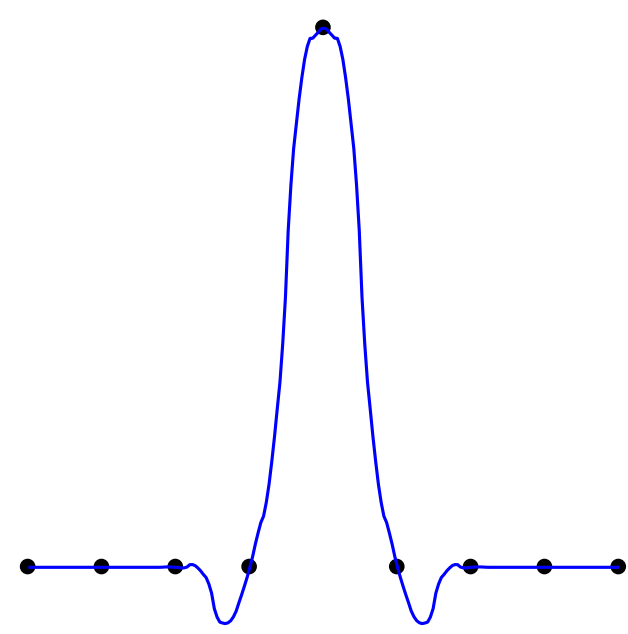

(b)

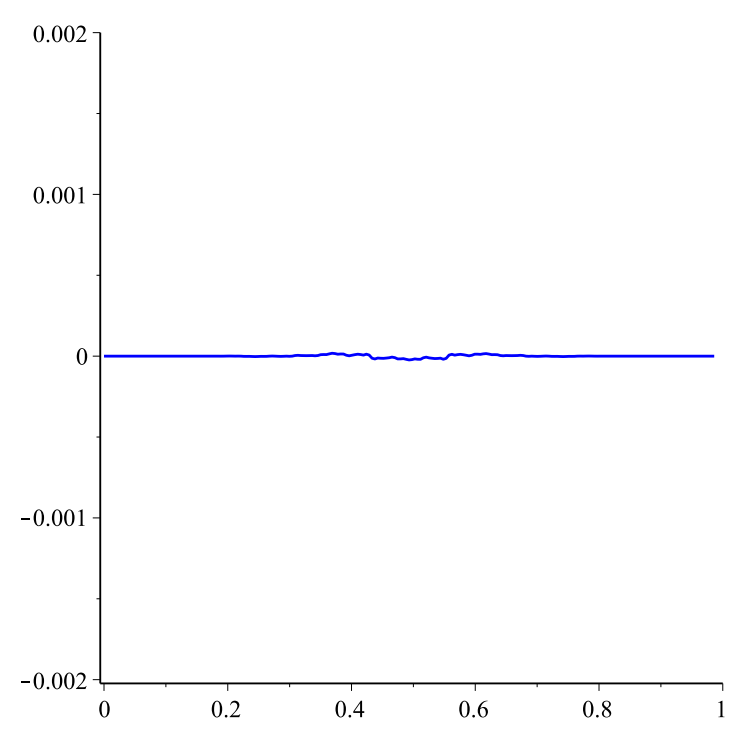

(c)

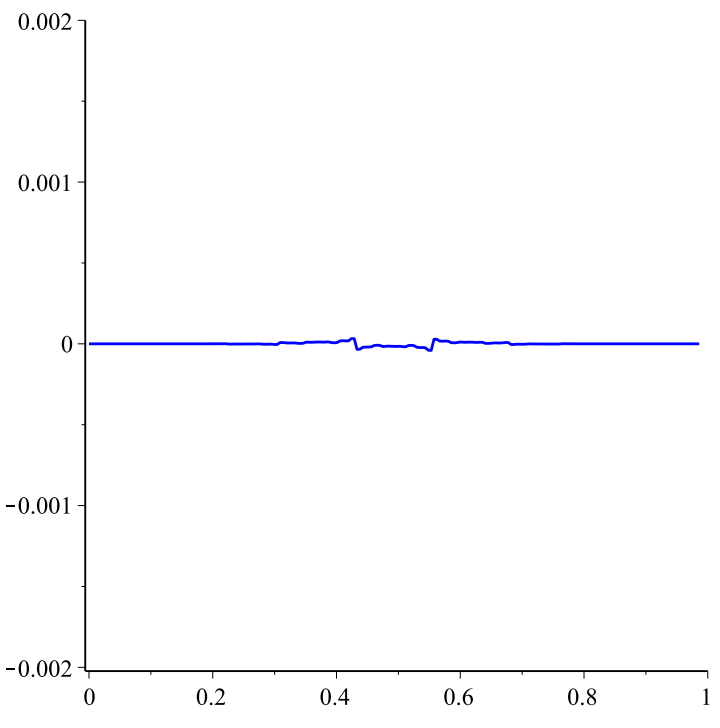

(d)

Figure 5. Results for various choices of the parameter $u$ are shown on the left together with their corresponding curvature on the right. From top to bottom: $u=0$ and 0.0074. (a) Limit curve at $u=0$; (b) Limit curve at $u=0.0074$; (c) Curvature plot of (a); (d) Curvature plot of (b). 


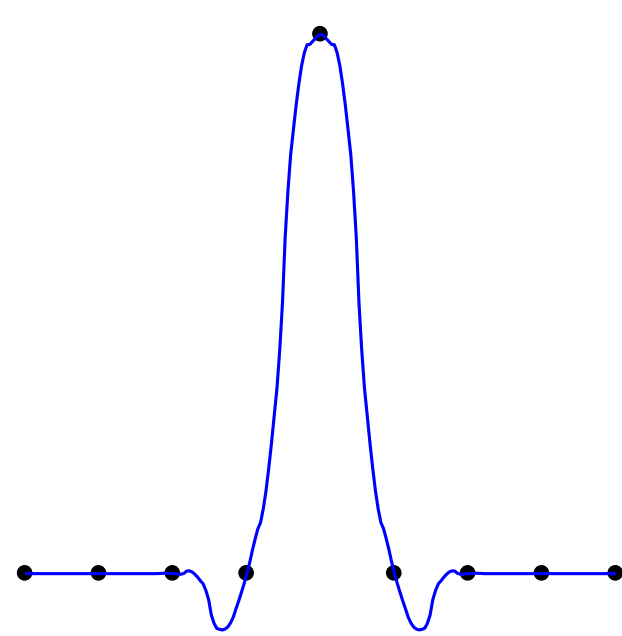

(a)

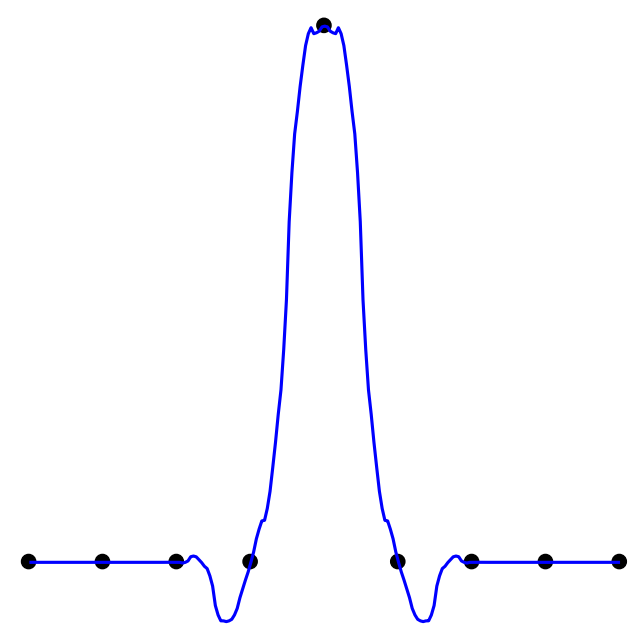

(b)

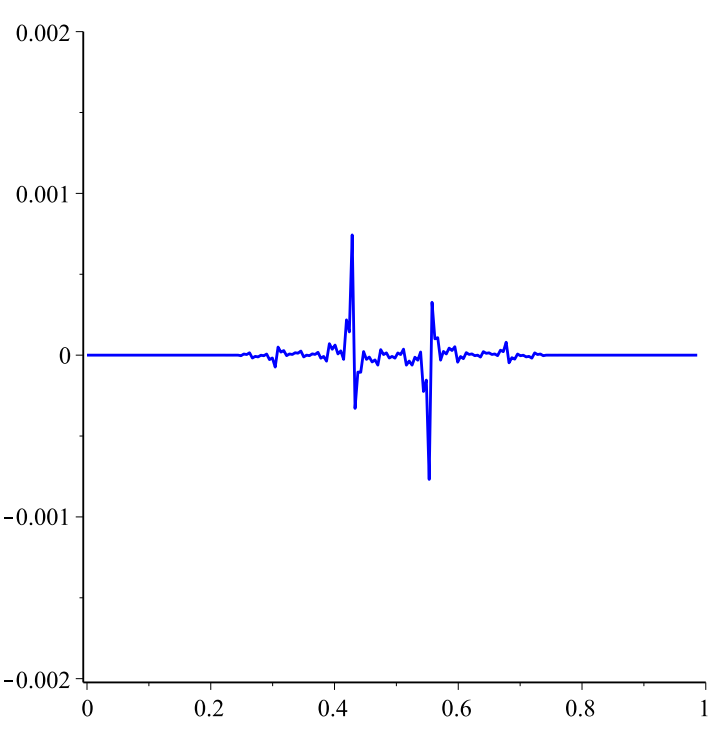

(c) Curvature plot of (a)

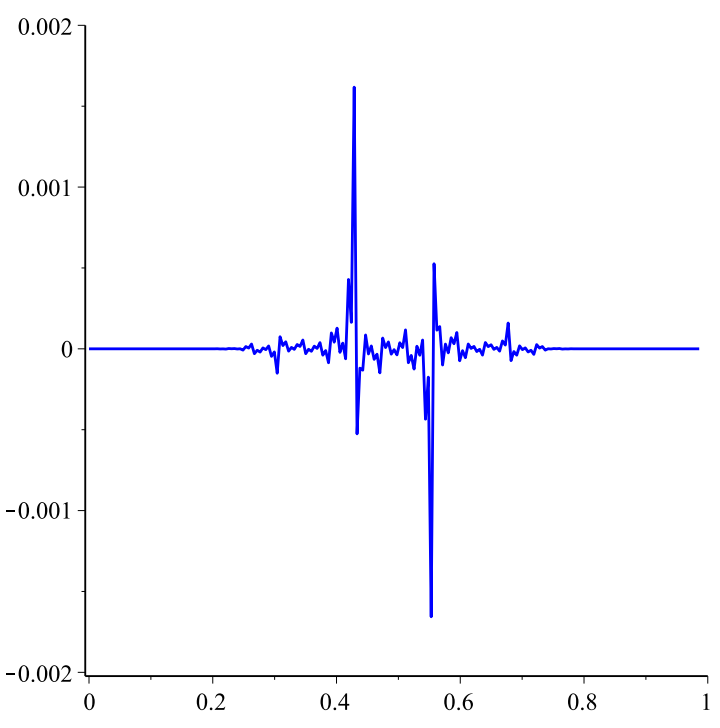

(d) Curvature plot of (b)

Figure 6. Results for various choices of the parameter $u$ are shown on the left together with their corresponding curvature on the right. From top to bottom: $u=0.0076$ and 0.0077. (a) Limit curve at $u=0.0076$; (b) Limit curve at $u=0.0077$; (c) Curvature plot of (a); (d) Curvature plot of (b).

\section{Numerical Examples and Conclusions}

In this section, we present some numerical examples to show monotonicity and convexity preserving behaviour of the FPR-scheme. At the end of the section, we discuss the conclusion of the work done so far.

\subsection{Numerical Examples}

We consider monotone data in Tables 1 and 2. Figure 7 explains monotonicity preserving property of the FPR-scheme. Figure 7a is generated by using monotone data as given in Table 1 . In this figure, dotted lines show the initial set of values and the solid line represents the limit curve generated by the $F P R$-scheme which is clearly monotonically increasing curve. Figure $7 \mathrm{~b}$ is generated by using monotone data as given in Table 2. In this figure, dotted lines show the initial set of values and the solid line represents the limit curve generated by the FPR-scheme, which is clearly monotonically increasing curve. 
Table 1. Monotone set of values.

\begin{tabular}{cccccccc}
\hline$x$ & 0 & 0.25 & 0.5 & 0.75 & 1 & 1.25 & 1.5 \\
\hline$y$ & 0 & 0.25 & 0.55 & 0.94 & 1.53 & 2.51 & 4.14 \\
\hline
\end{tabular}

Table 2. Monotone set of values.

\begin{tabular}{cccccccc}
\hline$x$ & -1.5 & -1 & -0.5 & 0 & 0.5 & 1 & 1.5 \\
\hline$y$ & -98.16 & -32 & -7.1 & 0 & 1.47 & 2 & 2.53 \\
\hline
\end{tabular}

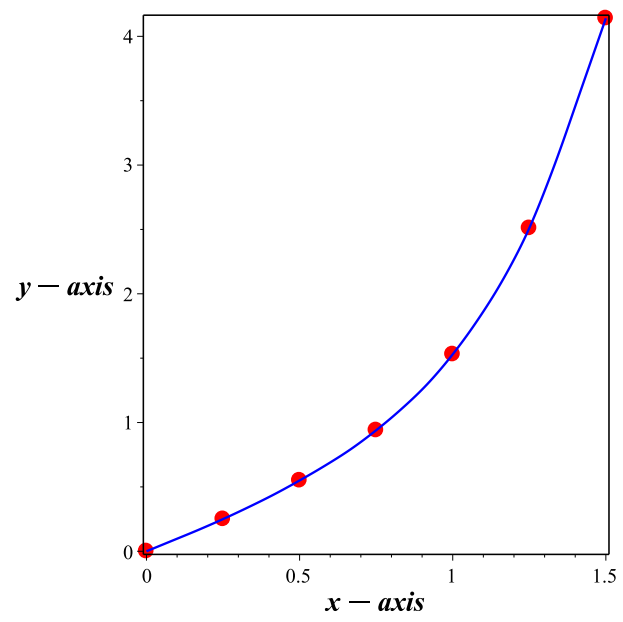

(a)

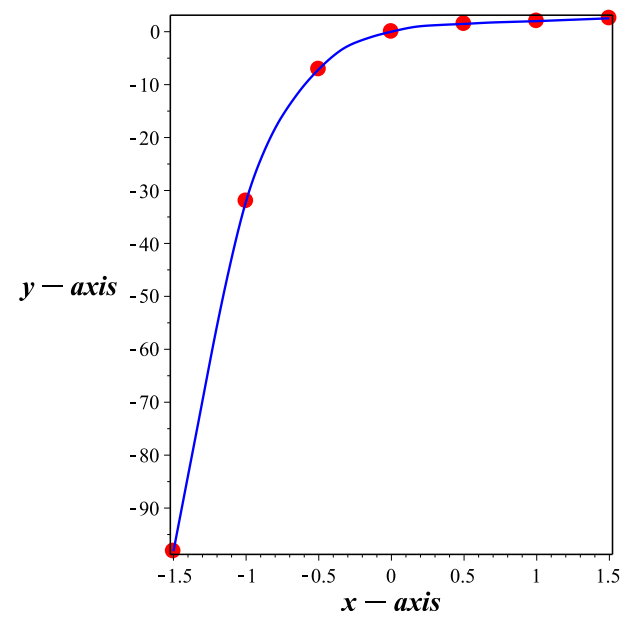

(b)

Figure 7. The monotone curves generated by the FPR-scheme (4). (a) Monotone curve at $u=0$ and (b) Monotone curve at $u=0.0076$.

We consider convex data in Tables 3 and 4. Figure 8 explains convexity preserving property of the FPR-scheme. Figure $8 \mathrm{a}$ is generated by using monotone data as given in Table 3. In this figure, dotted lines show the initial set of values and the solid line represents the limit curve generated by the FPR-scheme which is clearly monotonically increasing curve. Figure $8 \mathrm{~b}$ is generated by using monotone data as given in Table 4 . In this figure, dotted lines show the initial set of values and the solid line represents the limit curve generated by the FPR-scheme which is clearly monotonically increasing curve.

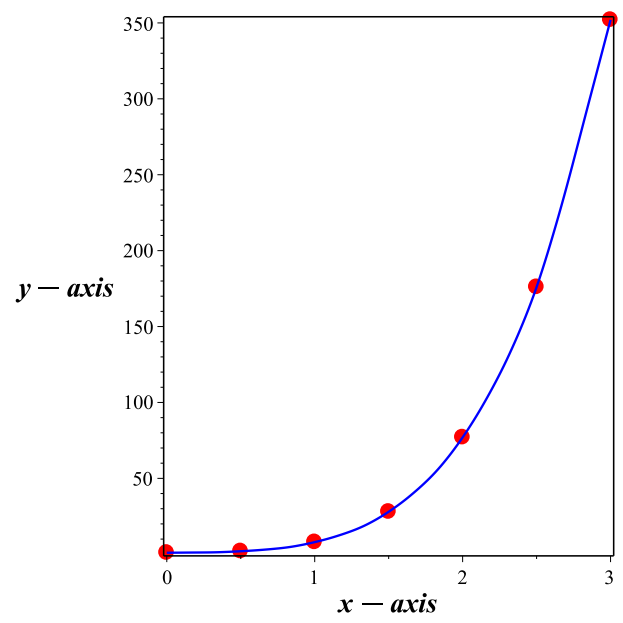

(a)

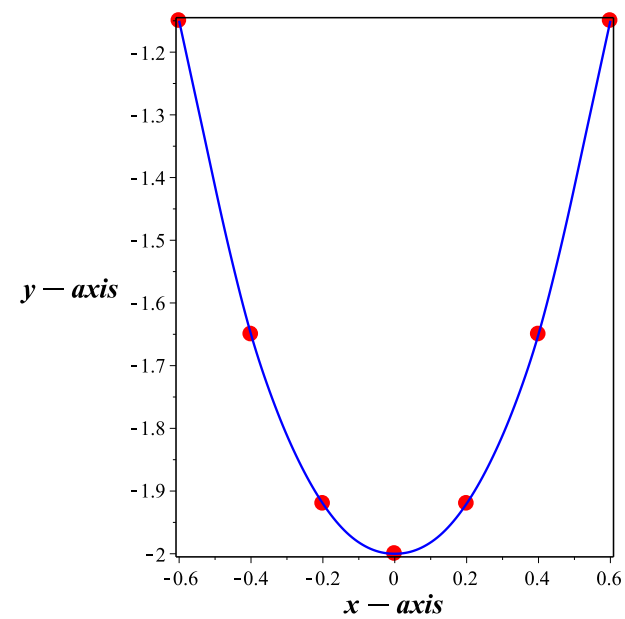

(b)

Figure 8. The convex curves generated by the FPR-scheme (4). (a) Convex curve at $u=0$ and (b) Convex curve at $u=0.0076$. 
Table 3. Convex set of values.

\begin{tabular}{cccccccc}
\hline$x$ & 0 & 0.5 & 1.0 & 1.5 & 2.0 & 2.5 & 3.0 \\
\hline$y$ & 1 & 2 & 8 & 28 & 77 & 176 & 352 \\
\hline
\end{tabular}

Table 4. Convex set of values.

\begin{tabular}{cccccccc}
\hline$x$ & -0.6 & -0.4 & -0.2 & 0 & 0.2 & 0.4 & 0.6 \\
\hline$y$ & -1.15 & -1.65 & -1.92 & -2 & -1.92 & -1.65 & -1.15 \\
\hline
\end{tabular}

\subsection{Conclusions}

In this paper, we have presented geometrical properties of the $F P R$-scheme, which improves on the scheme in various ways that meet different requirements. These geometric properties demonstrate that the shape-preservation of the limit curve is a useful mechanism for modifying the FPR-scheme. We have shown that by taking initial control data monotone and convex, the limit curves generated by the FPR-scheme are also monotone and convex. As observed, the FPR-scheme for various choice of shape control parameter can be considered more universal. Several examples are given which support our findings. Finally, the same idea could be applied to bivariate surfaces. A useful extension of this work is to analyze shape preserving behaviour of SSs when scattered data is considered.

Author Contributions: Conceptualization, P.A. and A.G.; formal analysis, D.B., K.S.N. and S.A.; methodology, D.B., K.S.N. and S. A.; software, P.A., B.N., A.G. and M.A.A.K.; supervision, D.B.; Writing-original draft, P.A., B.N. and A.G.; Writing-review \& editing, K.S.N., D.B. and S.A. All authors have read and agreed to the published version of the manuscript.

Funding: This research received no external funding.

Conflicts of Interest: The authors declare no conflict of interest.

\section{References}

1. Yadshalom, I. Monotonicity preserving subdivision schemes. J. Approx. Theory 1993, 74, 41-58. [CrossRef]

2. Carnicer, J.M.; Dahmen, W. Characterization of local strict convexity preserving interpolation methods by $C^{1}$ functions. J. Approx. Theory 1994, 77, 2-30. [CrossRef]

3. Dyn, N.; Kuijt, F.; Levin, D.; Van Damme, R. Convexity preservation of the four-point interpolatory subdivision scheme. Comput. Aided Geom. Des. 1999, 16, 789-792. [CrossRef]

4. Kuijt, F.; Van Damme, R. Shape preserving interpolatory subdivision schemes for non-uniform data. J. Approx. Theory 2002, 114, 1-32. [CrossRef]

5. Cai, Z. Convexity preservation of the interpolating four-point $C^{2}$ ternary stationary subdivision scheme. Comput. Aided Geom. Des. 2009, 26, 560-565. [CrossRef]

6. Hao, Y.X.; Wang, R.H.; Li, C.J. Analysis of a 6-point binary subdivision scheme. Appl. Math. Comput. 2011, 218, 3209-3216. [CrossRef]

7. Mustafa, G.; Deng, J.; Ashraf, P.; Rehman, N.A. The mask of odd points $n$-ary interpolating subdivision scheme. J. Appl. Math. 2012, 2012, 205863. [CrossRef]

8. Floater, M.; Beccari, C.; Cashman, T.; Romani, L. A smoothness criterion for monotonicity preserving subdivision. Adv. Comput. Math. 2013, 39, 193-204. [CrossRef]

9. Pitolli, F. Ternary shape-preserving subdivision schemes. Math. Comput. Simul. 2014, 106, 185-194. [CrossRef]

10. Mustafa, G.; Ashraf, P. A new 6-point ternary interpolating subdivision scheme and its differentiability. J. Inf. Comput. Sci. 2010, 5, 199-210. [CrossRef]

11. Siddiqi, S.S.; Noreen, T. Convexity preservation of six point $C^{2}$ interpolating subdivision scheme. Appl. Math. Comput. 2015, 265, 936-944. [CrossRef]

12. Akram, G.; Bibi, K.; Rehan, K.; Siddiqi, S.S. Shape preservation of 4-point interpolating non-stationary subdivision scheme. J. Comput. Appl. Math. 2017, 319, 480-492. [CrossRef]

13. Wang, Y.; Li, Z. A family of convexity-preserving subdivision schemes. J. Math. Res. Appl. 2017, 37, 489-495. 
14. Mustafa, G.; Bashir, R. Univariate approximating schemes and their non-tensor product generalization. Open Math. 2018, 16, 1501-1518. [CrossRef]

15. Novara, P.; Romani, L. On the interpolating 5-point ternary subdivision scheme: A revised proof of convexity-preservation and an application-oriented extension. Math. Comput. Simul. 2018, 147, 194-209. [CrossRef]

16. Asghar, M.; Iqbal, M. J; Mustafa, G. A family of high continuity subdivision schemes based on probability distribution. Mehran Univ. Res. J. Eng. Technol. 2019, 38, 389. [CrossRef]

17. Ghaffar, A.; Ullah, Z.; Bari, M.; Nisar, K.S.; Al-Qurashi, M.M.; Baleanu, D. A new class of 2m-point binary non-stationary subdivision schemes. Adv. Differ. Equ. 2019, 2019, 325. [CrossRef]

18. Zulkifli, N.A.B.; Karim, S.A.A.; Sarfraz, M.; Ghaffar, A.; Nisar, K.S. Image interpolation using a rational bi-cubic ball. Mathematics 2011, 7, 1045. [CrossRef]

19. Ashraf, P.; Sabir, M.; Ghaffar, A.; Nisar, K.S.; Khan, I. Shape-preservation of ternary four-point interpolating non-stationary subdivision scheme. Front. Phys. 2020, 7, 241. [CrossRef]

20. Ghaffar, A.; Ullah, Z.; Bari, M.; Nisar, K.S.; Baleanu, D. Family of odd point non-stationary subdivision schemes and their applications. Adv. Differ. Equ. 2019, 171, 1-20. [CrossRef]

21. Ghaffar, A.; Bari, M.; Ullah, Z.; Iqbal, M.; Nisar, K.S.; Baleanu, D. A new class of 2q-point non-stationary subdivision schemes and their applications. Mathematics 2017, 7, 639. [CrossRef]

22. Shahzad, A.; Khan, F.; Ghaffar, A.; Mustafa, G.; Nisar, K.S.; Baleanu, D. A novel numerical algorithm to estimate the subdivision depth of binary subdivision schemes. Symmetry 2020, 12, 66. [CrossRef]

23. Zou, L.; Song, L.; Wang, X.; Chen, Y.; Zhang, C.; Tang, C. Bivariate thiele-like rational interpolation continued fractions with parameters based on virtual points. Mathematics 2020, 8, 71. [CrossRef]

24. Peng, K.; Tan, J.; Li, Z.; Zhang, L. Fractal behavior of a ternary 4-point rational interpolation subdivision scheme. Math. Comput. Appl. 2018, 23, 65. [CrossRef]

25. Shang, Y. Lack of Gromov-hyperbolicity in small-world networks. Cent. Eur. J. Math. 2011, 10, 1152-1158. [CrossRef]

(C) 2020 by the authors. Licensee MDPI, Basel, Switzerland. This article is an open access article distributed under the terms and conditions of the Creative Commons Attribution (CC BY) license (http://creativecommons.org/licenses/by/4.0/). 